eISSN 2444-7986

DOI: https://doi.org/10.14201/orl201782.16057

Carta al Director

\title{
IX REUNIÓN NACIONAL DE LA COMISIÓN PARA LA DETECCIÓN PRECOZ DE LA HIPOACUSIA (CODEPEH)
}

\author{
IX National Meeting of the Commission for the Early Detection of \\ Hearing Loss (CODEPEH)
}

\author{
Rocío GONZÁLEZ-AGUADO \\ Hospital Marqués de Valdecilla. Santander. España. \\ Correspondencia: rocigonzagua@gmail.com
}

Fecha de recepción: 22 de abril de 2017

Fecha de aceptación: 22 de abril de 2017

Fecha de publicación: 23 de abril de 2017

Fecha de publicación del fascículo: 1 de junio de 2017

Conflicto de intereses: Los autores declaran no tener conflictos de intereses

Imágenes: Los autores declaran haber obtenido las imágenes con el permiso de los pacientes

Política de derechos y autoarchivo: se permite el autoarchivo de la versión post-print (SHERPA/RoMEO)

Licencia CC BY-NC-ND. Licencia Creative Commons Atribución-NoComercial-SinDerivar 4.0 Internacional

๔ Universidad de Salamanca. Su comercialización está sujeta al permiso del editor

\section{Señor Director:}

El pasado mes de septiembre tuvo lugar en Santander la IX reunión de la CODEPEH (Comisión para la Detección Precoz de la Hipoacusia infantil), en la cual se abordaron diferentes temas, teniendo vital importancia la etiología de la hipoacusia, así como al abordaje inter y multidisciplinar de esta patología.

En los dos días que compartimos asistimos a interesantes conferencias:

1. Diagnóstico etiológico de la sordera infantil. Gracias a la revolución en las técnicas de secuenciación, el diagnóstico genético es más accesible y nos vale para poder informar de forma correcta a los padres, sobre todo de cara a ofrecer un consejo genético, abriendo un futuro esperanzador a la terapia génica.

2. Aprendimos el manejo correcto, en base a la evidencia científica, de la otitis media secretora, dichas recomendaciones se publicaron posteriormente en una separata por parte de FIAPAS (Confederación española de familias de personas sordas; http://www.fiapas.es) [1].

3. Fuimos conscientes de que las indicaciones de los implantes cocleares han sufrido un drástico cambio en las últimas décadas. Siendo la única indicación en 1991, los adultos con hipoacusia bilateral (publicado en 1995 en el Documento de la Agencia de Evaluación de Tecnologías Sanitarias), pasamos en la 
década de los 2000 a implantar a niños (sabiendo que es la intervención más coste-efectiva que existe en base a calidad de vida). En la actualidad, la nueva indicación emergente es la de paciente con hipoacusia unilateral, ya que no se debe de olvidar la importancia de la binauralidad en la escucha con ruido de fondo.

4. Por último, nos presentaron nuevos avances en el campo de la física del sonido para poder mejorar la escucha con los dispositivos auditivos en entornos de ambiente ruidoso.

Aparte de las citadas conferencias se expusieron comunicaciones orales de diferentes temas e impartidas por varios profesionales, en las cuales nos quedó claro que para poder abordar de forma correcta e integral el tema de la hipoacusia neonatal deberemos crear equipos interdiscisciplinares, no multidisciplinares. Dichas comunicaciones orales son las que enviamos a modo de resumen de cada una de las mismas para ser publicadas en la revista.

Un cordial saludo.

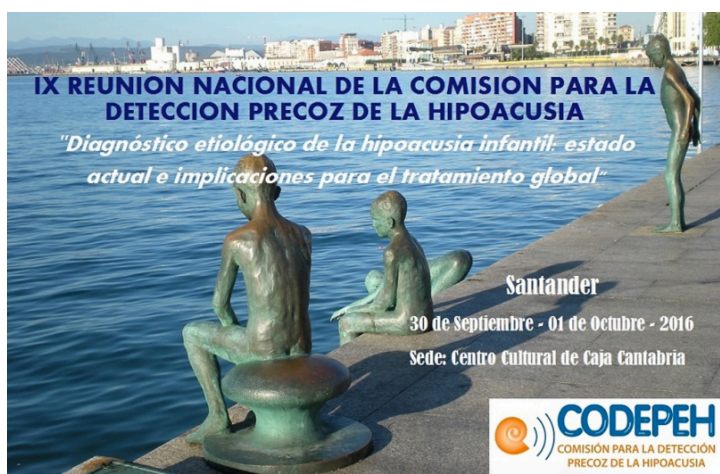

RESÚMENES DE COMUNICACIONES ORALES PRESENTADAS EN LA IX REUNIÓN DE LA CODEPEH (SANTANDER 30 DE SEPTIEMBRE - 1 DE OCTUBRE DE 2016)

ÍNDICE

1. Los padres como observadores de la calidad de vida de sus hijos implantados cocleares. (Publicado en Revista ORL [2])

2. Desarrollo del lenguaje en niños y niñas con detección temprana de la hipoacusia neonatal. (Publicado en Revista ORL [3])

3. Estudio analítico y observacional de las pruebas del lenguaje seleccionadas para niños/as con discapacidad auditiva.

4. Fiabilidad de los potenciales evocados auditivos de estado estable en la fase diagnóstica del cribado neonatal universal de la hipoacusia.

5. Etiología y evaluación audiológica de 183 neonatos diagnosticados de hipoacusia permanente. (Publicado en Revista ORL [4])

6. La calidad de vida y los factores influyentes en pacientes con Implante Coclear de 0 a 16 años en Cantabria.

7. Cribado auditivo neonatal periodo $2005-2016$ Hospital Universitario de Valme, Sevilla.

LOS PADRES COMO OBSERVADORES DE LA CALIDAD DE VIDA DE SUS HIJOS IMPLANTADOS COCLEARES.

Silvia Borkoski-Barreiro, Juan Carlos Falcón-González, Margarita Torres-García de Celis, Isabel Chicharro-Soria, Ángel Ramos-Macías.

Unidad de Hipoacusia. Servicio de Otorrinolaringología. Complejo Hospitalario Universitario Insular-Materno Infantil. Las Palmas de Gran Canaria. España.

Objetivo: El concepto de calidad de vida ha comenzado a utilizarse cada vez más en el campo de las evaluaciones en salud. Se ha empezado a investigar los efectos de la implantación a nivel del desarrollo social y emocional del niño. El objetivo es examinar los resultados de la salud relacionados con la calidad de vida en niños sordos implantados cocleares de entre 11 y 12 años de edad y sus padres. Material y Método: Estudio observacional, descriptivo transversal en 24 niños prelocutivos implantados unilaterales o bilaterales sucesivos de 11 y 12 años de edad y sus padres. Utilizando el Cuestionario de calidad de vida pediátrica PedsQL TM (Versión 4.0- español para España), para medir la salud relacionados con la calidad de vida, completado de forma independiente por niños y padres respectivamente. Resultados: Mediante la T-Student los resultados de niños y padres en las diferentes escalas evaluadas observamos que en las escalas de funcionamiento emocional y académica es donde se obtienen resultados estadísticamente significativos $(p<0,05)$. Al comparar el resultado total del Cuestionario con las diferentes escalas evaluadas, en todas ellas se obtienen resultados estadísticamente significativos $(p<0,05)$. Conclusiones: Los resultados del estudio muestran que los niños disfrutan de los beneficios del uso de su implante coclear. Ellos reportan beneficios en todas las áreas estudiadas (física, emocional, social y académica). Los padres son periodistas válidos sobre el estado de la calidad de vida global de sus hijos ya que dieron respuestas similares a las dadas por sus hijos 
DESARROLLO DEL LENGUAJE EN NIÑOS Y NIÑAS CON DETECCIÓN TEMPRANA DE LA HIPOACUSIA NEONATAL.

José Ignacio Benito-Orejas; Rosa Belén Santiago-Pardo; Carmen Romero-Ureña; Ángel Luis Sánchez-Rosso; M Fe Muñoz-Moreno; Marina Escarda-Bolaños; Ana HerreroGaliacho; Celia González-Núñez; Ma Isabel Rico-Paino Rebeca Álvarez-Mielgo; Lidia Moreno; María Conde; Miriam González; Jorge Gutiérrez; Cristina Arroyo; Rocío Cerrejón; Alberto Martín; Lucía Isabel de Castro; Aarón García; María Fernández; Cristina García.

Hospital Clínico Universitario de Valladolid. Facultad de Educación y Escuela de Logopedia de la Facultad de Medicina de la Universidad de Valladolid.

Introducción y objetivo: Dado que el potencial discapacitante que causa la deficiencia auditiva neonatal disminuye con una detección e intervención tempranas, hemos elaborado un proyecto de investigación, con el que nos proponemos conocer el grado de desarrollo del lenguaje de los niños y niñas que procedentes del cribado auditivo universal, han sido diagnosticados/as por nosotros de hipoacusia prelingual en estos últimos 15 años y analizar las variables determinantes y las que son modificables. Material y método: Partimos de los datos almacenados durante este tiempo, donde se incluye a 282 niños/as con hipoacusia. Hemos tenido la oportunidad de crear un grupo de trabajo en el que coincidimos especialistas de la audición infantil y del lenguaje, por lo que contamos con medios suficientes para el estudio. Resultados: Describimos los elementos que configuran este proyecto de investigación, en relación al equipo de trabajo y a su desarrollo. Tras aplicar unos criterios de exclusión/inclusión, hemos seleccionado a un grupo de unos 45 niños/as entre 3 y 15 años, definiendo sus características auditivas. Mediante pruebas específicas adaptadas a la edad, estudiaremos los diferentes aspectos del lenguaje; y a través de una entrevista estructurada realizada a los padres, intentaremos determinar las variables que influyen en el proceso re-habilitador Finalmente, los datos serán analizados estadísticamente. Conclusiones: El programa de cribado auditivo universal nos ha permitido una intervención más precoz, lo que debería mejorar los niveles de lenguaje de los niños/as detectados/as con hipoacusia. Aunque el desarrollo normalizado de la comunicación depende de otros factores difíciles de determinar, a través del protocolo presentado pretendemos equiparar estos resultados, validando el proceso de cribado/diagnóstico e intervención de nuestro medio.

ESTUDIO ANALITICO Y OBSERVACIONAL DE LAS PRUEBAS DEL LENGUAJE SELECCIONADAS PARA NIÑOS/AS CON DISCAPACIDAD AUDITIVA.

$M^{a}$ Isabel Rico-Paino; Belén Santiago-Pardo'; José Ignacio Benito-Orejas ${ }^{2}$; Ángel Luis Sánchez-Rosso ${ }^{3}$; Ana Herrero-Galiacho; Celia González-Núñez; Carmen Romero-Ureña ${ }^{1}$.

Departamento de Pedagogía de la Facultad de Educación ${ }^{1}$. Servicio de Otorrinolaringología del Hospital Clínico Universitario ${ }^{2}$. Departamento de Psicología y Titulación de Logopedia de la Facultad de Medicina ${ }^{3}$. Valladolid. España.
Introducción y objetivo: Dentro del proyecto de investigación interdisciplinar iniciado en 2015 en la Universidad de Valladolid sobre el "Desarrollo del lenguaje en niños/as con hipoacusia neonata» cuya detección temprana se hubiera realizado en el Hospital Clínico Universitario, uno de los objetivos fundamentales consistió en seleccionar aquellas pruebas que mejor permitieran estudiar desde un punto de vista formal, los diferentes aspectos del lenguaje del niño/a con hipoacusia. La escasez de publicaciones científicas sobre la valoración de estas pruebas cuando se utilizan en sujetos con hipoacusia, nos ha permitido conocer las características y ámbito de aplicación de las que hemos utilizado, concediéndonos la licencia de complementar los resultados con nuestra opinión. Material y Método: Las pruebas de lenguaje escogidas y analizadas han sido: el test PEABODY; el registro fonológico de Laura Bosch; EDAF; EMLE-TALE 2000; PLON-R y BLOC-SR. En función de los resultados de cada prueba, obtenemos unos porcentajes de realización en centiles y en rangos de edad, que nos permiten apreciar los posibles retrasos. Esta evaluación ha sido desarrollada por estudiantes de logopedia y logopedas recién graduados, de los que hemos querido conocer sus impresiones mediante un cuestionario dirigido, complementando estos resultados con la opinión de expertos logopedas en el terreno práctico. Resultados: En términos generales todas las pruebas han resultado adecuadas en función de su finalidad. Las que mejor se corrigen son EDAF, PEABODY y BLOC-SR y la que peor, el registro fonológico de LauraBosch. Para nosotros, el procedimiento de aplicación de PEABODY Y PLON-R es ideal para niños con hipoacusia, presentando mayores dificultades el EDAF y BLOC-SR y siendo muy difíciles de aplicar, el registro fonológico de Laura Bosch y el EMLE. Conclusiones: Consideramos que las pruebas seleccionadas son suficientes para evaluar el desarrollo del lenguaje en el niño/a con deficiencia auditiva, siempre y cuando las apliquemos correctamente en función de la edad de desarrollo y tengamos en cuenta las modificaciones específicas que debemos adoptar en esta población.

FIABILIDAD DE LOS POTENCIALES EVOCADOS AUDITIVOS DE ESTADO ESTABLE EN LA FASE DIAGNÓSTICA DEL CRIBADO NEONATAL UNIVERSAL DE LA HIPOACUSIA.

Patricia García-Cabo ${ }^{1}$; Laura Fernández-Vañes ${ }^{1}$; Faustino Núñez-Batalla ${ }^{2}$; Sabel Noriega-Iglesias ${ }^{3}$; Maite GuntínGarciá; Pilar Carro Fernández ${ }^{2}$; Jose Luis LlorentePendás ${ }^{1}$

${ }^{1}$ Servicio de ORL, Hospital Universitario Central de Asturias (HUCA), Oviedo, España. ${ }^{2}$ Unidad de Hipoacusia Infantil, Servicio de ORL, Hospital Universitario Central de Asturias, (HUCA), Oviedo, España. ${ }^{3}$ Facultad de Psicología y Logopedia, Universidad de Oviedo, Oviedo, España. ${ }^{4}$ Programa de Atención al Déficit Auditivo Infantil del Principado de Asturias, IATYS, Fundación Vinjoy, Oviedo, España.

Introducción y objetivo: La audiometría convencional es el patrón de referencia para cuantificar y describir una hipoacusia. Sin embargo, se precisan métodos alternativos para los pacientes muy jóvenes que no pueden responder con fiabilidad. Los potenciales evocados auditivos de tronco (PEATC) es el método más extendido para determinar de forma objetiva los umbrales auditivos, sin embargo, no informan específicamente en 
cada frecuencia. La llegada de los potenciales evocados de estado estable (PEAEE) permite conseguir una determinación más específica en frecuencias. El presente trabajo describe y compara los umbrales obtenidos mediante PEATC, PEAEE y audiometría convencional en un grupo de niños que presentan varios grados de pérdida auditiva. Material y Método: Se llevó a cabo una comparación entre los umbrales de PEATC, PEAEE y audiométricos obtenidos de 35 niños detectados en el programa de cribado auditivo neonatal. Resultados: La diferencia media $( \pm D E)$ entre los umbrales de los PEATC y la banda de $4.000 \mathrm{~Hz}$ de los PEAEE fueron $11,2 \mathrm{~dB}( \pm 13)$ para el oído derecho y $10,2 \mathrm{~dB}( \pm 11)$ para el izquierdo. Las correlaciones de Pearson entre los umbrales de los PEAEE y audiométricos fue 0,80 y $0,91(500 \mathrm{~Hz}) ; 0,84$ y $0,82(1.000 \mathrm{~Hz}) ; 0,85$ y $0,84(2.000 \mathrm{~Hz}) ; 0,83$ y $0,82(4.000$ $\mathrm{Hz}$ ), respectivamente para oído derecho e izquierdo. Conclusiones: La técnica de los PEAEE es una técnica a tener en cuenta para su inclusión en la batería de test que se emplea en el diagnóstico audiológico de los niños con hipoacusia.

ETIOLOGÍA Y EVALUACIÓN AUDIOLÓGICA DE 183 NEONATOS DIAGNOSTICADOS DE HIPOACUSIA PERMANENTE.

José Ignacio Benito-Orejas; Beatriz Ramírez-Cano; Marleny Casasola-Girón; Ana Sánchez-Martínez; Andrea Cifuentes-Navas; Darío Morais-Pérez.

Servicio de ORL y PCF. Hospital Clínico Universitario de Valladolid.

Introducción y objetivo: La hipoacusia neonatal es una de las discapacidades más frecuentes, con importantes consecuencias para el niño y su familia durante toda la vida. El desarrollo del cribado auditivo universal y el avance de la medicina molecular, de la genética y de la neurociencia han perfeccionado el diagnóstico precoz de la hipoacusia infantil y consecuentemente su intervención. Con este trabajo queremos mostrar los aspectos audiológicos y las causas de las hipoacusias permanentes diagnosticadas durante estos últimos 20 años. Material y Método: Se revisan retrospectivamente los registros de los niños diagnosticados con menos de 3 años de edad de hipoacusia permanente, durante el periodo 1994-2015, en un hospital de tercer nivel. Evaluamos el momento de inicio, lateralidad, tipo y grado de hipoacusia. En función de los antecedentes, pruebas genéticas y otras exploraciones complementarias, presentamos los resultados de nuestro estudio diagnóstico. Resultados: Teniendo en cuenta que sólo valoramos a niños con menos de 3 años de edad, un $87 \%$ de las hipoacusias permanentes $>30 \mathrm{~dB} \mathrm{HL}$ se diagnostican al nacimiento (congénitas) y un $13 \%$ de forma tardía. Sus principales características son la bilateralidad $(81 \%)$, el predominio neurosensorial (85\%) y el grado profundo (42\%) o moderado (30\%), más prevalente en las formas unilaterales. En cuanto al diagnóstico etiológico, un $47 \%$ son de origen genético $(29 \%$ de las cuales son sindrómicas), un $25 \%$ de causa adquirida y un $28 \%$ desconocida. Conclusiones: Nuestros resultados concuerdan en términos generales con los refrendados en la literatura. Consideramos fundamental el seguimiento de un protocolo consensuado que oriente en el proceso diagnóstico.
LA CALIDAD DE VIDA Y LOS FACTORES INFLUYENTES EN PACIENTES CON IMPLANTE COCLEAR DE 0 A 16 AÑOS EN CANTABRIA.

Andrea Martín-Pérez; Laura Callejo-Cuadrillero.

Servicio Universitario de Investigación Gimbernat. Cantabria. España.

Introducción: Según la Organización Mundial de la Salud un niño sordo es aquel cuya agudeza auditiva resulta insuficiente para permitirle desarrollar la lengua oral propia de su entorno, participar en las actividades normales de su edad y seguir la enseñanza escolar normalizada. El Implante Coclear es un aparato que transforma los sonidos y ruidos del medio ambiente en energía eléctrica capaz de actuar sobre el nervio coclear, desencadenando una sensación auditiva, pero sin restablecer la audición normal en un individuo sordo.

Objetivos: El objetivo del presente estudio es realizar un análisis de cómo influye la rehabilitación especializada en la calidad de vida de personas con Implante Coclear, además de obtener información sobre las adaptaciones escolares y las ayudas recibidas. Material y Metodología: Mediante este estudio observacional, descriptivo y transversal, se ha analizado la calidad de vida de niños con Implante Coclear de 0-16 años de Cantabria, utilizando un cuestionario realizado por los autores del presente estudio. Resultados: Participaron 13 personas, la mayoría fueron implantados entre el año o los dos años de vida, comenzando la rehabilitación tras la activación del Implante Coclear, aunque la frecuencia semanal y la duración en meses son muy variables. En general, reciben apoyos educativos y ayudas económicas que mejoran la calidad de vida. Conclusiones: En este estudio observacional se determina que la calidad de vida se ve influenciada positivamente por una implantación precoz seguida de una intervención especializada mantenida en el tiempo. Una vez escolarizados la inclusión social se ve favorecida por los apoyos a nivel educativo. Todo ello está relacionado con la información y las ayudas que reciben las familias.

CRIBADO AUDITIVO NEONATAL PERIODO 2005-2016 HOSPITAL UNIVERSITARIO DE VALME, SEVILLA.

Juan Solanellas-Soler; Juan Miguel Jiménez-Morales; Isabel Reyes-Tejero, Ulises Bidón-Gómez.

Hospital Universitario de Valme. Sevilla. España.

Introducción y objetivo: El protocolo de detección de hipoacusias en el recién nacido del Hospital Universitario de Valme contempla tres fases consecutivas para el diagnóstico. Las dos primeras son de cribaje y la tercera es de diagnóstico. Durante el periodo comprendido entre 2005 y 2016 se han utilizado de manera conjunta y/o secuencial las dos pruebas de cribado para el primer y segundo filtro: Las otoemisiones acústicas transitorias (OEAt) y los potenciales evocados auditivos automáticos (PEATCa). El objetivo principal de dicha práctica ha sido de obtener una amplia cobertura universal. Otros objetivos específicos han consistido en reducir de manera significativa el número de niños que deben ser reevaluados en un segundo filtro, disminuir el número de familias a las que se genera una ansiedad ante la posibilidad de que su bebé pueda padecer una sordera al no pasar dicha prueba, evitar posibles pérdidas de sujetos a los que una vez realizado el primer filtro son recitados 
para el segundo filtro y que no acuden por causas diversas (pérdidas del Programa) y concentrar el esfuerzo del cribado en el primer filtro para evitar recitaciones posteriores que inciden en un costo económico y de personal innecesario. Material y Método: La práctica ha consistido en la realización de OEA a todos los recién nacidos que no tienen indicadores de riesgo de hipoacusia en el momento del nacimiento. En el caso de que no pasaran la prueba, inmediatamente, a continuación, y antes de ser dados de alta, se les practicaron los PEATCa. En caso de que presentaran un indicador de riesgo se efectuaron PEATCa. El segundo filtro se basó exclusivamente en la realización de PEATCa. Finalmente, el tercer filtro (diagnóstico) fue efectuado mediante potenciales evocados auditivos convencionales (PEATC). Resultados: El número de recién nacidos en el Hospital Universitario de Valme durante el periodo comprendido entre el 1 de enero de 2005 y el 30 de junio de 2016 ha sido de 39925 bebés. Se ha realizado el primer filtro a 38849 lo que ha supuesto una cobertura del $98,97 \% .582$ recién nacidos no pasaron el primer filtro lo que supone un $1,49 \%$ de derivación a un segundo filtro. El grado de cobertura de dicha fase ha sido de un $93,81 \%$ (546 niños). Finalmente, del total de 112 bebés que no pasaron el segundo filtro se realizaron PEATC en una tercera fase a 101 niños (cobertura del 90,18\%). El total de niños diagnosticados de hipoacusia durante este periodo de tiempo ha sido de 44 pacientes $(1,47 \times 1000)$. Conclusiones: Los indicadores del Hospital Universitario de Valme en relación a las tres fases del cribado de hipoacusia en el recién nacido alcanzan unos valores que se enmarcan dentro de las normas de calidad óptima del cribado de hipoacusia en el recién nacido. Se aconseja en hospitales como el nuestro, con una alta incidencia de nacimientos, la realización de ambas pruebas OEA y PEATCa como práctica que contribuye a disminuir falsas alarmas a las familias de recién nacidos y pérdidas de bebés que no acuden a un segundo filtro.

\section{BIBLIOGRAFÍA}

1. CODEPEH (Faustino Núñez, Carmen Jáudenes, José Miguel Sequí, Ana Vivanco, José Zubicaray). Diagnóstico y tratamiento de la otitis media secretora infantil: recomendaciones CODEPEH 2016. Revista FIAPAS (separata). 2016;159:I-XXIII. Disponible en: http://www.bibliotecafiapas.es/pdf/REVI STA\%20159.pdf.[Citado el 23-04-2017].
2. Borkoski-Barreiro S, Falcón-González J, Torres-García-de-Celís M, ChicharroSoria I, Ramos-Macías Á. Los padres como observadores de la calidad de vida de sus hijos implantados cocleares. Revista ORL. 2017;8(2):105-10. Disponible en: http://revistas.usal.es/index.php/2444-7986/article/view/15215. [Citado 24 Abr 2017].

3. Benito-Orejas J, Santiago-Pardo R, Romero-Ureña C, Sánchez-Rosso Á, Muñoz-Moreno $M$, Escarda-Bolaños $M$, et al. Metodología aplicada en el estudio del desarrollo del lenguaje en niños con detección temprana de la hipoacusia neonatal. Revista ORL. 2017;8(2):85103. Disponible en: http://revistas.usal.es/index.php/2444-7986/article/view/15133. [Citado 24 Abr 2017].

4. Benito-Orejas J, Ramírez-Cano B, Casasola-Girón $M$, Sánchez-Martínez $A$, Cifuentes-Navas V, Morais-Pérez D. Etiología de la hipoacusia infantil. Revista ORL. 2017;8(2):69-83. Disponible en: http://revistas.usal.es/index.php/2444-7986/article/view/15088. [Citado 24 Abr 2017].

\section{OTRAS REFERENCIAS}

Web de la IX Reunión Nacional de la CODEPEH (Santander 30 septiembre al 1 de octubre de 2016). [Citado 24 Abr 2017].

FIAPAS (Confederación española de familias de personas sordas). http://www.fiapas.es/FIAPAS/index.html [Citado 24 Abr 2017]. 